\title{
PERIGOS NO USO DE AGROTÓXICOS PELA SAÚDE PÚBLICA NO CONTROLE VETORIAL DO AEDES AEGYPTI (PERIGOS NO USO DE AGROTÓXICOS PELA SAÚDE PÚBLICA)
}

\author{
DANGER IN THE USE OF AGROTOXICS FOR PUBLIC HEALTH IN VECTOR CONTROL \\ (DANGER IN THE USE OF AGROTOXICS FOR PUBLIC HEALTH)
}

Thayná Rhayssa Batista da SILVA¹, Polyana Felipe Ferreira da COSTA², Solange Laurentino dos SANTOS ${ }^{3}$.

Palavras-chave:

Pesticidas;

Toxicidade;

Arbovírus;

Controle de vetores;

Aedes ha.

Keywords:

Pesticides;

Toxicity;

Arboviruses;

Vector control;

Aedes.

\begin{abstract}
RESU M O
O controle das arboviroses dengue, chinkugunya e síndrome congênita do zika vírus tem mantido a aplicação de agrotóxicos como principal estratégia de controle do mosquito Aedes aegypti. Este estudo analisa criticamente a utilização de agrotóxicos pela saúde pública no controle da dengue mediante revisão bibliográfica, documental e de artigos científicos de acordo com as seguintes categorias: (1) as condições de vida e vulnerabilidades; (2) Abordagens de controle do Aedes aegypti no Brasil; (3) a vigilância em saúde pública; e, o (4) (des) conhecimento sobre os perigos do uso de agrotóxicos. O controle vetorial do Aedes aegypti utiliza agrotóxicos dos grupos químicos organofosforados, carbamatos e piretróides cuja exposição pode gerar efeitos agudos e crônicos na saúde humana. É importante 0 conhecimento da população sobre a toxicidade e os riscos à saúde.
\end{abstract}

\section{A B S T R A C T}

The control of arboviruses maintains the application of chemical insecticides as the main strategy to control Aedes aegypti. This study critically analyzes the use of pesticides by public health in the control of dengue using the bibliographic, documentary and scientific articles review according to the following categories: (1) living conditions and vulnerabilities; (2) Aedes aegypti control approaches in Brazil; (3) Public health surveillance; (4) (un) knowledge about the dangers of the use of pesticides. The vector control of the Aedes aegypti uses pesticides from organophosphate, carbamate and pyrethroid chemical groups, whose exposure can generate acute and chronic effects on human health. The population's knowledge of toxicity and health risks is important.

\footnotetext{
${ }^{1}$ Mestre em Saúde Coletiva pelo Programa de Pós-Graduação em Saúde Coletiva da Universidade Federal de Pernambuco. Email: thaynarbio@gmail.com.

2 Professora da Universidade de Pernambuco (UPE) e Mestre em Saúde Coletiva pelo Programa de Pós-Graduação em Saúde Coletiva da Universidade Federal de Pernambuco. E-mail: polyanafelipe@yahoo.com.br.

3 Professora Associada do Centro de Ciências Médicas da Universidade Federal de Pernambuco. E-mail: solaurentino@hotmail.com.
} 


\section{INTRODUÇÃO}

O controle de Aedes aegypti (Linaeus, 1762) é um desafio para a saúde pública em nível mundial devido à sua relação com a atual situação de transmissão das arboviroses Dengue, Febre Chikungunya (CHIKF), Síndrome Congênita do Zika Vírus (SCZV) e Febre Amarela Urbana (WORLD HEALTH ORGANIZATION, 2019). É considerado um dos mais importantes insetos hematófagos entre todos os Arthropoda, pois durante o seu hematofagismo, realizado pelas fêmeas infectadas pode transmitir agentes biológicos, como vírus, bactérias e helmintos que em determinadas condições produz doenças e epidemias (NEVES, 2016). A Organização Mundial de Saúde (OMS) aponta que para obter um controle efetivo, localmente adaptado e sustentável, a ação deve ser focada em quatro pilares fundamentais destacando 0 fortalecimento da colaboração com outros setores fora da saúde, melhorar a vigilância e monitoramento, ampliação e integração das ferramentas de controle de vetores e engajamento de comunidades (WORLD HEALTH ORGANIZATION, 2019). O cenário epidemiológico brasileiro das arboviroses é preocupante pela ocorrência da tríplice epidemia - Dengue, CHIKF e SCZV - até a Semana Epidemiológica 12 para o ano de 2019, foram registrados 290.889 casos prováveis da tríplice epidemia, e ainda sob risco da reemergência de Febre Amarela Urbana (BRASIL, 2019). Todas essas arboviroses têm em comum A. aegypti como vetor, bem como a possibilidade de outros vetores participarem da cadeia de transmissão, como Aedes albopictus (Skuse 1894), do dengue (CENTER FOR DISEASE CONTROL AND PREVENTION. 2020) e o Culex quinquefasciatus (Say, 1823) do vírus Zika; (GUEDES et al., 2017), o que dificulta ainda mais o processo de determinação nos contextos urbanos, principalmente, nas cidades onde as condições sanitárias são precárias.

Os problemas decorridos por essas doenças têm levado a população a demandar por ações rápidas das autoridades de saúde suscitando estratégias de controle vetorial. Há um consenso de que os sistemas de vigilância precisam reformular seus modelos de monitoramento, controle e avaliação frente a essa realidade e, de que o controle da dengue como problema de saúde pública pode ser alcançado pela integração do controle de vetores com as vacinas (ACHEE et al., 2015). Por outro lado, no Brasil, a persistência de um modelo centrado na eliminação ou diminuição da densidade de vetores mediante uso de tecnologias biocidas é uma realidade imposta em detrimento da problemática do saneamento ambiental (AUGUSTO: SANTOS; DIDERICHSEN, 2016).

Tendo como referência as estratégias adotadas pelo Programa Nacional de Controle da Dengue (PNCD), busca-se no presente artigo analisar criticamente o uso de agrotóxico no controle de Aedes aegypti e as consequências na saúde humana. 


\section{MÉTODO}

Trata-se de uma pesquisa documental, entendida como o procedimento que se utiliza de métodos e técnicas para apreensão, compreensão e análise de variados tipos de documentos (SÁ-SILVA; ALMEIDA; GUINDANI, 2009). Neste caso, buscou-se a explicação do problema a partir de referenciais teóricos publicados em documentos que abordaram aspectos do uso de agrotóxicos utilizados no controle do A. aegypt. Foi realizado um levantamento nos principais documentos oficiais do Ministério da Saúde, Organização Pan-americana de Saúde e Organização Mundial de Saúde. A pesquisa foi complementada com busca em artigos científicos, dissertações, teses e livros. O material coletado neste estudo ficou limitado aos idiomas português, inglês e espanhol. Não sendo estabelecida nenhuma restrição ao período das publicações.

Foi realizada análise preliminar e de conteúdo para identificação das substâncias utilizadas seguida da sistematização de acordo com as seguintes categorias: (1) A interdependência das condições de vida em que vivem classes sociais mais vulneradas; (2) Abordagens de controle do Aedes aegypti no Brasil; (3) 0 olhar da vigilância em saúde pública; e o (4) (des) conhecimento sobre os perigos do uso de agrotóxicos. A realização desse estudo foi de setembro de 2016 a março de 2017.

Esta pesquisa utilizou dados secundários de domínio público. Não necessitou de anuência institucional e faz parte da dissertação de mestrado aprovada pelo Comitê de Ética em Pesquisa da Universidade Federal de Pernambuco - UFPE (CAEE nº 64635017.9.0000.5208).

\section{RESULTADOS E DISCUSSÃO}

\subsection{A interdependência das condições de vida em que vivem classes sociais mais vulneradas}

Tão urgente quanto a reforma política, a reforma urbana têm sido alvo de debates pela séria crise de saúde pública causada pela infestação do mosquito A. aegypti, considerado o principal vetor de doenças em seres humanos no mundo e transmissor do vírus zika (ZIKV) e chikungunya (CHIKV), além dos vírus causadores da Dengue (DENV-1, DENV-2, DENV-3 e DENV-4) e da febre amarela urbana (MARICATO, 2016).

Para entender o que nos faz conviver com esse mosquito há décadas, se faz necessário um olhar mais ampliado para além da saúde pública. A situação nas periferias brasileiras onde são enfrentados sérios problemas na área do saneamento: água, esgoto, drenagem de águas pluviais e coleta de resíduos sólidos são condições perfeitas para a produção de vetores de doenças (COSTA, 2016), tornando-se possível entender que certos grupos populacionais são mais susceptíveis do que outros para contrair determinadas 
doenças e são influenciados pela condição ambiental. Estudo realizado na cidade do Recife mostrou a correlação negativa estatisticamente significante entre a coleta de lixo e a incidência de dengue, demonstrando que a coleta de lixo pode impactar negativativamente nos casos de dengue (SOBRAL; SOBRAL, 2019). Neste sentido, concordamos com a importância de que ações nas condições sanitárias poderiam ser adotadas como estratégias de prevenção pelos municípios.

Segundo Maricato (2016), o saneamento básico seria fundamental para o controle do mosquito. E a segregação espacial da pobreza seria central nessa determinação. Em estudo de Santos et al., (2015) os técnicos municipais reconhecem que a transmissão da dengue sobre influência dos condicionantes sociais e que interesses econômicos geram desigualdades na forma de ocupação do espaço urbano. Consideramos que as iniquidades sociais influenciam diretamente na disseminação de vetores como $0 \mathrm{~A}$. aegypti e 0 discurso oficial em que a população é responsabilizada por proliferar criadouros em suas residências é extremamente inadequado.

\subsection{Abordagens de controle do Aedes aegypti no Brasil para prevenção das arboviroses}

O combate ao A. aegypti foi institucionalizado no Brasil, de forma sistematizada, a partir do século vinte, com o objetivo de reduzir o número de casos da febre amarela urbana, que havia levado milhares de pessoas a óbito (BRASIL, 1942). Entre o período de 1902 a 1907, em que Oswaldo Cruz foi diretor geral de saúde pública, foram instituídas brigadas sanitárias com a finalidade de detectar casos de febre amarela e eliminar focos do mosquito. Entre os anos de 1928 e 1929 iniciou no município do Rio de Janeiro uma epidemia que alcançou os demais municípios do estado, com registros de 738 casos e 478 óbitos (FRANCO, 1969).

Medidas de controle foram adotadas nas Américas com o incentivo da Fundação Rockefeller nas décadas de 1930 e 1940. Em 1947, a Organização Pan-Americana de Saúde (OPAS) filiada a OMS coordenaram a erradicação do vetor no continente, por intermédio do Programa de Erradicação do A. aegypti. Eficientes programas foram implementados em todos os países latino-americanos, entre o final da década de 1940 e o início de 1950. O Brasil, assim como outros países latinos americanos, participou da campanha de erradicação continental e teve êxito na primeira eliminação do vetor em 1955. Essa espécie foi eliminada em quase toda a América, com exceção dos Estados Unidos, Suriname, Venezuela, Cuba, Jamaica, Haiti, República Dominicana e uma pequena parte da Colômbia (BRAGA; VALLE, 2007; LOWY,1999).

Entre 1955 e 1973, o A. aegypti foi considerado erradicado no Brasil, contudo em 1976 foi reintroduzido, tanto por falhas na vigilância epidemiológica como pelo crescimento populacional acelerado em decorrência do intenso fluxo migratório das zonas rurais para. A partir de então, o vetor é encontrado em 
todos os estados brasileiros, principalmente nas regiões mais quentes como as regiões nordeste, norte e parte do sudeste, tornando seu controle uma tarefa desafiante para as autoridades de saúde (TAUIL, 2001).

Em 1990, foi criada a Fundação Nacional de Saúde (Funasa) com o objetivo de fomentar 0 saneamento básico à população e passou a ser responsável pela coordenação das ações de controle da dengue. Nesta década, observou-se a difusão do A. aegypti e o agravamento do quadro epidemiológico. A rápida dispersão do vetor, a partir do ano de 1994, propiciou a circulação viral em maior número de Estados e Municípios culminando com o que se denominou de terceira onda epidêmica da doença em 1997-1998 (BRAGA; VALLE, 2007).

No ano de 1996, o Ministério da saúde elaborou o Plano de Erradicação do Aedes aegypti (PEAa), no intuito de reduzir os casos de dengue hemorrágica. O plano, que previa um modelo descentralizado com a participação das três esferas do governo não alcançou o êxito esperado e ponderou a inviabilidade técnica de erradicação do mosquito no curto e médio prazo (FUNASA, 1996).

Em julho de 2001, a Funasa resignou a meta de erradicar o A. aegypti e passou a considerar 0 controle vetorial, implementando o Plano de Intensificação das Ações de Controle da Dengue (PIACD), que preconizava ações em municípios. Em 2002, foi implantado o Programa Nacional de Controle da Dengue (PNCD), dando continuidade a algumas propostas do PIACD e enfatizando a necessidade de mudanças nos modelos anteriores (FUNASA, 2001a, 2002). Santos e Augusto (2005) apontam que paralelamente a essa condição de agravamento da doença e, dos vários planos de controle implementados, o órgão responsável pelo controle de endemias, caminhava no sentido da descentralização das ações desde 1990, observando-se dificuldades das instâncias regionais e locais para assumirem as ações de controle. Esse fato pode ter atuado como força motriz para o descontrole vetorial e o ressurgimento da endemia, além de outros condicionantes como enorme êxodo rural e o crescimento sem precedentes das periferias urbanas.

Mesmo diante dos vários planos de controle do vetor da Dengue elaborados pelo Ministério da Saúde, a utilização de agrotóxicos para o controle é dominante na saúde pública há mais de 30 anos. A transmissão da doença se mantém e os quatro sorotipos (DENV-1, DENV-2, DENV-3 e DENV-4) continuam circulando nas diversas regiões do país. Atualmente, as abordagens de controle vetorial difundidas englobam medidas de controle mecânico, biológico e químico, sendo o último grupo mais utilizado, demonstrando uma tradição sanitária no controle vetorial "químico dependente" (BRASIL, 2016).

Os problemas decorridos pela tríplice epidemia e ainda sob risco de uma quarta, pela possibilidade de reemergência de Febre Amarela na sua incidência urbana, somada as recorrentes epidemias de dengue há várias décadas justificam a demanda da população às autoridades de saúde por ações rápidas e efetivas para o controle vetorial. Entretanto a adoção da estratégia de controle das epidemias baseada no controle químico do vetor, apesar de sua baixa efetividade observada pelas recorrentes epidemias das arboviroses e 
de microcefalia merecem uma avalição crítica desse modelo de controle (ABRASCO, 2016; BRITO; TEIXEIRA, 2017; CAMPOS et al., 2018; SOUZA et al., 2018).

O uso de agrotóxicos para controle de populações do A. aegypti na sua fase adulta (adulticidas) e na sua forma larvária (larvicidas) pode ser feito por meio do "tratamento" focal perifocal - segundo Santos (2003, p. 56) o emprego do termo tratamento merece uma crítica ao ser comparado ao termo empregado na medicina clínica para o ato de curar - e, de aspersão aeroespacial de inseticidas em Ultra Baixo Volume (UBV) (FUNASA, 2001b). O Ministério da Saúde utiliza agrotóxicos recomendados pela OMS, que o faz por meio do World Health Organization Pesticides Evaluation Scheme (WHOPES) (WHOPES, 2010), programa que desde 1960 serve como referência para muitos no uso e manejo de produtos contra insetos de importância em saúde pública. Na Nota Técnica de n. ${ }^{0}$ 109/2010 de Combate à Dengue e observada a recomendação da intensificação do uso da UBV motorizada e costal no domicílio a nas vias públicas (BRASIL, 2010).

Atualmente, o WHOPES recomenda diferentes princípios ativos para o controle do A. aegypti nas fases larvária e adulta (WHOPES, 2010).

O Quadro 1, apresenta as substâncias utilizadas contra larvas recomendados para uso em água potável. Destacamos a recomendação do uso do pyriproxifen que se baseia em revisão da literatura disponível no Guidelines for Drinking Water Quality- Intenational Programe on Chemical Safety - GDWQ/IPCS e considera o produto seguro para uso no controle do vetor A. aegypti, inclusive em água de consumo humano. Essa recomendação explícita de segurança e da baixa toxicidade do produto não leva em consideração as necessidades das comunidades de garantia da qualidade da potabilidade da água de beber (BRASIL, 2014) principalmente, em lugares onde existe uma grave situação de desabastecimento de água.

Quadro 1 Larvicidas preconizados pela Organização Mundial da Saúde para uso em água potável no controle do vetor da Dengue, segundo grupo químico, ação e classe toxicológica.

\begin{tabular}{|c|c|c|c|}
\hline Agrotóxico & Grupo químico & Ação & Classe toxicológica \\
\hline Diflubenzuron & Benzoilureas & Regulador do Desenvolvimento & $\mathrm{IV}$ \\
\hline Novaluron & Benzoilureas & Regulador do Desenvolvimento & $\mathrm{IV}$ \\
\hline Piriproxifen & Éter piridiloxipropílico & Regulador do Desenvolvimento & $\mathrm{IV}$ \\
\hline Spinosad & Espinosina & Neurotóxico & $\mathrm{III}$ \\
\hline Temefós & Organofosforado & Neurotóxico & $\mathrm{III}$ \\
\hline Fenthion & Organofosforado & Neurotóxico & $\mathrm{II}$ \\
\hline Pirimiphos-methy & Organofosforado & Neurotóxico & $\mathrm{II}$ \\
\hline Chlorpyrifos & Organofosforado & Neurotóxico & $\mathrm{II}$ \\
\hline
\end{tabular}

Fonte: Site Direitos Urbanos, 2015. 
No Quadro 2 estão apresentados os produtos utilizados contra os insetos adultos, nestes grupos destacamos a utilização do organofosforado da classe toxicológica II - Malathion - , que é considerado pela Agência Internacional de Pesquisa em Câncer (IARC) como potencialmente cancerígeno para os seres humanos (GUYTON et al., 2015).

Quadro 2 Inseticidas contra mosquitos adultos e para aplicação espacial recomendados pela Organização Mundial de Saúde para aplicação residual segundo grupo químico, ação e classe toxicológica e espaços abertos.

\begin{tabular}{|c|c|c|c|}
\hline \multicolumn{4}{|c|}{ PRODUTOS USO CONTRA INSETOS ADULTOS } \\
\hline Agrotóxico & Grupo químico & Ação & Classe toxicológica \\
\hline Alfacipermetrina & Piretróide & Neurotóxico & II \\
\hline Bendiocarb & Carbamato & Inibidores de acetilcolinesterase & 1 \\
\hline Bifenthin & Piretróide & Moduladores de canais de sódio & III \\
\hline Cyfluthrin & Piretróide & Moduladores de canais de sódio & ॥ \\
\hline Deltametrin & Piretróide & Neurotóxico & III \\
\hline Etofenprox & Piretróide & Moduladores de canais de sódio & IV \\
\hline Fenitrothion & Organofosforado & Neurotóxico & III \\
\hline Lambda-cialotrin & Piretróide & Neurotóxico & III \\
\hline Propoxur & Carbamato & Inibidores de acetilcolinesterase & ॥ \\
\hline Malathion & Organofosforado & Neurotóxico & ॥ \\
\hline Pirimiphos-metil & Organofosforado & Inibidores de acetilcolinesterase & III \\
\hline \multicolumn{4}{|c|}{ INSETICIDAS PARA APLICAÇÃO ESPACIAL } \\
\hline Deltametrina & Piretróide & Neurotóxico & III \\
\hline Lambda-cialotrin & Piretróide & Neurotóxico & III \\
\hline Malathion & Organofosforado & Neurotóxico & II \\
\hline d,d-trans-cyphenothrin & Piretróide & Moduladores de canais de sódio & III \\
\hline
\end{tabular}

Fonte: BRASIL 2014. 
Sabe-se que a utilização de agrotóxicos no controle vetorial como uma das principais estratégias não é apenas utilizada pelo setor público, mas também pelo setor privado como no uso doméstico (SANTOS et al., 2007). Infelizmente, tais práticas não têm se mostrado efetivas e se percebe o aumento no número de localidades infestadas pelo vetor, com resistência aos agrotóxicos passíveis de uso na saúde pública.

A baixa efetividade das ações de controle do A. aegypti vem sendo justificada pelas autoridades sanitárias como de responsabilidade individual da população e da gestão municipal (VALLE, 2015). Porém as práticas com enfoque maior nas ações de combate ao vetor alicerçado no uso de produtos químicos, sem que se considerem os riscos à saúde e ao ambiente em detrimento às ações de saneamento ambiental e melhoria das condições sanitárias dos lugares vem sendo questionada por diversos órgãos de pesquisa e de saúde (ABRASCO, 2016).

\subsection{O olhar da vigilância em saúde pública}

Em 1990, foi estimado pela OMS, o acontecimento de três milhões casos de intoxicação, dos quais 75 mil desenvolveram câncer por exposição e considerando que há subnotificações, o número real é ainda maior, além do baixo número de atendimentos hospitalares e diagnóstico preciso, tendo em vista que os sintomas não são patognomônicos, ou seja, são sintomas bastante gerais (OPAS, 1996). Segundo esse documento, após absorvidos por contato direto (manuseio do produto) ou indireto (água contaminada e alimentos ingeridos), cujo acesso ao corpo é via transdérmica, inalatória ou digestiva, os agrotóxicos podem suscitar diversos efeitos na saúde humana, sendo eles de forma aguda, subaguda ou crônica:

- Aguda: Os primeiros sintomas aparecem pouco tempo após a exposição de produtos com alto grau de toxicidade. Podendo ocorrer de forma leve, moderada ou grave, dependerá da quantidade de agrotóxico absorvido pelo indivíduo. Os sinais e sintomas são translúcidos e objetivos variando de acordo com o ingrediente ativo (IA), como: náuseas, vômitos, fraqueza, cefaléia, dispnéia, desmaio, convulsões, contrações musculares, epistaxe.

- Subaguda: Acontece por uma desproteção pequena ou moderada a produtos com médio ou alto grau de toxicidade, possuindo um aparecimento de sintomas mais lento. São subjetivos e vagos, tais como mal-estar, fraqueza, cefaléia, sonolência, epigastralgia, entre outros;

- Crônica: Possui um surgimento tardio, após meses ou anos, através de uma exposição pequena ou moderada a um ou vários químicos, fomentando danos irreversíveis, como efeitos neurotóxicos retardados, lesões renais e hepáticas, paralisias, neoplasias, alterações cromossomiais, teratogênese, desregulações endócrinas, entre outros. Na maioria das vezes são confundidos com outros distúrbios ou até mesmo nunca são associados ao agente causador. 
À medida que conhecemos os casos de doenças associadas aos efeitos crônicos acarretados pelos agrotóxicos, existem grandes obstáculos em obtermos dados confiáveis. Essas associações estão bem apontadas por estudos toxicológicos, clínicos e epidemiológicos de exposição a agrotóxicos e diferentes efeitos crônicos desses biocidas no trabalho de Rigoto e Aguiar (2015), onde são revelados os distúrbios endócrinos; efeitos sobre a reprodução; alterações imunológicas, que repercutem em cânceres; malformações congênitas; doenças neurológicas, hepáticas, renais; etc. As autoras apontam vários questionamentos que não estão respondidos de forma satisfatória, invisibilizando esses agravos no campo científico e social. No sistema nervoso, pode provocar alterações neurocomportamentais, encefalopatias ou até mesmo as tentativas de suicídio como foi observado por Pires, Caldas e Recena, (2005) no seu estudo em que relacionou a prevalência das tentativas de suicídio à exposição aos organofosforados monocrotofós e metamidofós.

Também são relatadas alterações na reprodução humana, em que cerca de $70 \%$ das mulheres que moram em cidades onde há uso constante de agrotóxico relataram perda de feto e 39,4\% revelaram ter perdido um filho com menos de um ano de vida (IARC, 2015). Além desses fatores, apresentaram-se as questões de parto prematuro, recém-nascido de baixo peso, malformações congênitas, infertilidade masculina, associadas aos efeitos de desregulação endócrina e imunogenética de alguns ingredientes ativos como agrotóxicos (ARAÚJO; NOGUEIRA; AUGUSTO, 2000; CARLSEN et al., 1992; GRISOLIA, 2005).

Em um estudo sobre os riscos de carcinogênese química através de uma análise teórica utilizando os modernos métodos da química quântica, Pavão e Leão (LEÃO; PAVÃO, 1996) sinalizaram que:

Alguns compostos utilizados no combate ao Aedes aegypti apresentaram um forte caráter eletrofilico, uma das características de agentes químicos carcinogênicos. Uma análise estatística levando em consideração cinco descritores da ação carcinogênica mostrou malathion, fenitrotion e o temefós com elevada atividade, comparável a carcinógenos conhecidos, como aflatoxina, benzopireno, tetracloreto de carbono.

Neste estudo teórico os autores apresentam os dados com base em determinadas propriedades químicas do malathion, feniltrotion e temefós ao comparar com outras substâncias carcinogênicas. No círculo maior apresentado na Figura 1, nota-se essa afinidade entre os químicos utilizados comparando-os com os grupos de carcinógenos conhecidos, não- carcinógenos (etanol e água) e protetores (vitamina $\mathrm{C}$, resveratrol, AAS). Com base neste estudo os autores apontaram um alerta e a recomendação de não utilizar tais substâncias por serem potencialmente perigosas. 


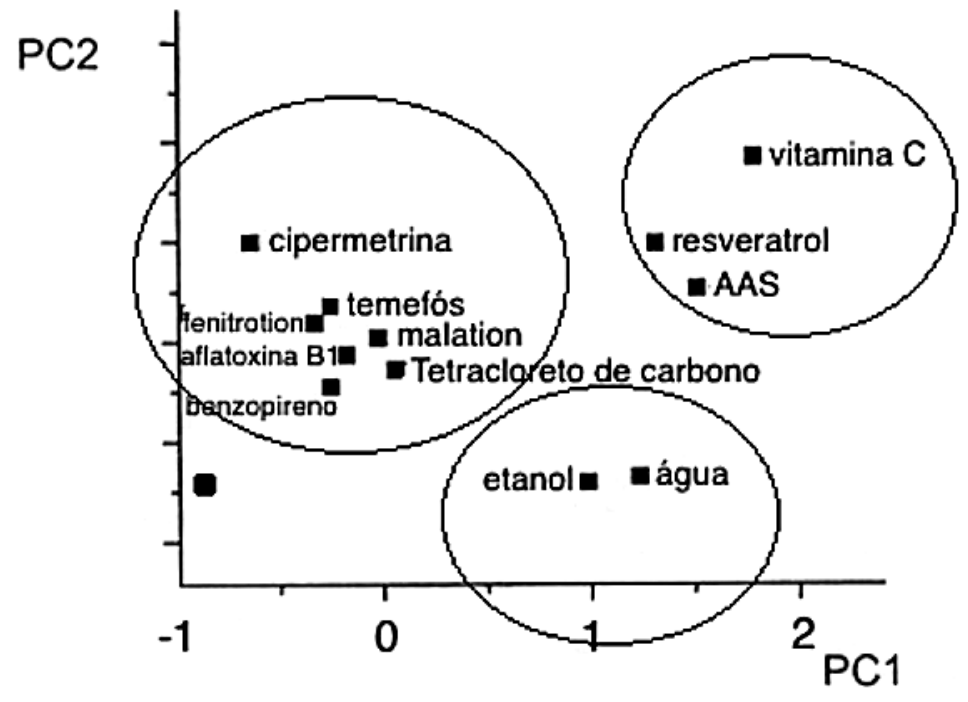

Figura 1 Agrupamento de carcinógenos, não-carcinógenos e protetores conforme PC1 (primeira componente principal os parâmetros EA - afinidade eletrônica - e $\Delta \mathrm{Hf}$ - calor de formação - apresentam maior peso na discriminação dos compostos) e PC2 (segunda componente principal - em que o $\Delta q$ - diferença das cargas positivas de átomos presentes no composto e a carga negativa, $q=-0,32$ do 06 da guanina).

Em estudo de revisão sistemática de literatura em que foram avaliados 83 artigos científicos, com 0 objetivo de investigar o uso de agrotóxicos e a ocorrência de câncer nos anos 1992 a 2003 . A conclusão do autor foi de que vários estudos mostravam associação entre a exposição aos agrotóxicos e a incidência de neoplasias, tais como leucemia e linfoma não - Hodgkin, e, em menor grau, existiu uma associação entre os agrotóxicos e alguns tumores sólidos, como os de cérebro e próstata (BASSI, 2007). Isso demonstra que esses agrotóxicos têm a potencialidade de provocar danos celulares ou inibir o sistema de supressão das mutações genéticas dos organismos interrompendo uma cadeia de reações alteradas, podendo ser o ponto de partida para a evolução de diversos tipos de câncer (RIGOTO; AGUIAR, 2015).

Além da extensa literatura científica que confirma as relações entre os agrotóxicos e as neoplasias do sistema hematopoiético, nos últimos anos estão sendo condensadas evidências sobre as relações entre esses químicos e neoplasias em diversas localizações do organismo, como cânceres de pulmão, estômago, melanoma, próstata, cérebro, testículos e sarcomas (FONTENELE et al., 2010; ROMANO et al., 2008; SOLOMON; SCHETTLER, 2000).

Essas evidências conduziram o Instituto Nacional de Câncer (INCA) a lançar uma Nota Pública em abril de 2015, objetivando "[...] demarcar o posicionamento do INCA contra as atuais práticas de uso de agrotóxicos no Brasil e ressaltar seus riscos à saúde, em especial nas causas do câncer" (BRASIL, 2015).

Destaca-se também que em março de 2015 a IARC publicou a Monografia volume 112, em que, após a avaliação da carcinogenicidade de cinco ingredientes ativos de agrotóxicos por uma equipe de pesquisadores de 11 países, incluindo o Brasil, classificou como prováveis agentes carcinogênicos para 
humanos (Grupo 2A), os inseticidas malathion e diazinon e os inseticidas tetraclorvinfós e parathion como possíveis agentes carcinogênicos para humanos (Grupo 2B). Enfatiza-se que a malathion é autorizado e amplamente usado no território brasileiro como inseticida em campanhas de saúde pública no controle vetorial do Aedes aegypty (GUYTON et al., 2015; IARC, 2015).

\section{Desregulação endócrina}

Interferente endócrino (IE) trata-se de uma substância química que pode interferir no funcionamento natural do sistema endócrino de espécies animais, incluindo os seres humanos e que mesmo presente em concentrações extremamente baixa, é capaz de interferir no funcionamento natural do sistema endócrino. Os principais sistemas afetados pelos IEs são: imunológico, nervoso e reprodutor (GHISELLI; JARDIM, 2007).

Fontenele et al. (2010) cita, como exemplo de interferente endócrino, agrotóxicos registrados no Brasil e utilizados no controle vetorial associados à desregulação endócrina como por exemplo a cipermetrina e malathion. Friedrich (2013) afirma que esses agrotóxicos estão relacionados a efeitos como:

Agonismo ou antagonismo das funções dos receptores de estrógenos e andrógenos, desregulação do eixo hormonal hipotálamo-pituitária, inibição ou indução de prolactina, progesterona, insulina, glicocorticoides, tireoideanos e indução ou inibição da enzima aromatase, que é responsável pela conversão do precursor andrógeno em estrógenos.

Dentre tantos pesquisadores brasileiros que trazem os efeitos à saúde causada pelos agrotóxicos, Gurgel (2005), em seu estudo sobre os Agentes de Saúde Pública em Pernambuco, já trazia como resultado evidências que dentre os entrevistados da sua pesquisa 47\% apresentam distúrbios da visão; $38 \%$ alergias; $34 \%$ dorsalgias; $25 \%$ cefaleia frequente; $24 \%$ infecções com mais frequência do que as pessoas do seu convívio; $20 \%$ (71) apresentam náuseas frequentes; $19 \%$ distúrbio do sono; $19 \%$ distúrbio da audição; 18\% má digestão frequentemente; $17 \%$ parestesias; e 17\% tremores nas mãos.

O Quadro 3 mostra uma sistematização dos efeitos à saúde humana e os órgãos afetados decorrentes da utilização de agrotóxicos. Essa sistematização foi baseada em revisão de literatura, base de dados científicos, documentos de instituições reguladas e de pesquisas dentre elas a IARC, ANVISA, ABRASCO, FIOCRUZ e Agência de Proteção Ambiental dos Estados Unidos. 
Quadro 3 Efeitos de agrotóxicos na saúde humana por órgão ou sistemas

\begin{tabular}{|c|c|}
\hline ÓRGÃO/SISTEMA & EFEITOS NO ORGANISMO \\
\hline Sistema Nervoso & $\begin{array}{c}\text { Asteno-vegetativa, Autismo depressão, ansiedade, transtorno do sono, polineurite, } \\
\text { radiculite, neuropatias encefalopatia, distonia vascular, esclerose cerebral, neurite } \\
\text { retrobulbar, angiopatia da retina, fasciculações, tremores, convulsões, tonteiras, } \\
\text { cefaleias, Mal de Alzheimer, de consciência/coma. }\end{array}$ \\
\hline Sistema Respiratório & $\begin{array}{c}\text { Traqueíte crônica, pneumofibrose, enfisema pulmonar, asma brônquica, irritação } \\
\text { laringotraqueal. }\end{array}$ \\
\hline Sistema Cardiovascular & $\begin{array}{c}\text { Miocardite tóxica crônica, insuficiência coronária crônica, hipertensão, hipotensão, } \\
\text { doenças vasculares. }\end{array}$ \\
\hline Trato Gastrointestinal & $\begin{array}{c}\text { Gastrite crônica, duodenite, úlcera, colite crônica (hemorrágica, espástica, formações } \\
\text { polipóides), hipersecreção e hiperacidez gástrica, prejuízo da motricidade. }\end{array}$ \\
\hline Fígado & Hepatite crônica, colecistite, insuficiência hepática. \\
\hline Sistema Imunológico & Anemia aplástica, câncer. \\
\hline Aparelho Urinário & Diurese frequente e involuntária. \\
\hline Rins & Albuminúria, nictúria, alteração do clearance da uréia, nitrogênio e creatinina. \\
\hline Sistema Reprodutor & Disfunção reprodutiva. \\
\hline Sistema Hematopoético & Leucopenia, eosinopenia, monocitose, alterações na hemoglobina. \\
\hline Pele & Dermatites, eczemas. \\
\hline Olhos & Conjuntivite, síndrome colinérgica, lacrimejamento, dor ocular, visão prejudicada, \\
\hline
\end{tabular}

Fonte: Elaborado pelas autoras, 2016.

Além dos efeitos tóxicos evidentes descritos na literatura científica nacional e internacional, as ações para o enfrentamento do uso dos agrotóxicos têm como base o Direito Humano à Alimentação Adequada DHAA - (previsto nos artigos $6^{\circ}$ e $27^{\circ}$ da Constituição da República Federativa do Brasil de 1988), a Política Nacional de Segurança Alimentar e Nutricional (Decreto $n^{0} 7.272$, de 25/08/2010), a Política Nacional de Saúde Integral das Populações do Campo e da Floresta - PNSIPCF (Portaria $n^{0} 2.866$ de 02/12/2011), a Política Nacional de Saúde do Trabalhador e da Trabalhadora (Portaria $n^{0} 1.823$, de 23/08/2012) e a Política Nacional de Agroecologia e Produção Orgânica - PNAPO (Decreto nº 7.794, de 20/08/2012) (FONTENELE et al., 2010). 


\section{4 (Des)conhecimento do perigo sobre a utilização do agrotóxico}

Lefevre et al., (2007), apontam, em sua pesquisa, para a necessidade de se estudar a problemática educativa a fim de identificar, com mais precisão e detalhe, o conhecimento da população, seus eventuais lapsos, seu grau de organicidade, com vistas ao enfrentamento da defasagem entre a informação e a prática, o que poderá contribuir para o incremento da desejável e necessária participação popular no combate ao vetor

Estudo realizado por Chiaravalloti et al., (2002) demonstrou que, isoladamente, os conhecimentos sobre o vetor em discussão e as arboviroses transmitidas pelo mesmo, foram incorporados pela população, mas não corresponderam necessariamente a uma mudança de hábitos e consequentemente a uma redução dos criadouros. Estudo de Santos, Cabral e Augusto (2011) observou que há um conhecimento, principalmente, sobre as características do vetor, entretanto há desconhecimento da população sobre 0 larvicida químico utilizado na água utilizada para consumo doméstico e para beber.

Entretanto, a forma reducionista como são apresentadas as soluções para o controle das arboviroses e do seu principal vetor, o mosquito A. aegypti, não permite que as pessoas reflitam sobre os condicionantes socioambientais presentes em seus contextos de vida, bem como sobre os riscos à saúde que lhes são impostos ao se utilizar de substâncias químicas para o controle do mosquito, em detrimento de outras formas de redução de criadouros como o saneamento ambiental e o abastecimento adequado e água.

Mas partindo da compreensão de que o ciclo de vida do vetor é, essencialmente, ligado à água, já que a postura e o desenvolvimento dos ovos ocorrem predominantemente em recipientes deixados com água exposta ou parada. É que se questiona a ação dos gestores de saúde pública quando não discutem com a população os riscos à saúde do uso de agrotóxicos nos ambientes domiciliares e peridomiciliares. Os dados apresentados ao longo do artigo permitem perceber a invisibilidade sobre os perigos decorrentes da utilização indiscriminada de agrotóxicos na saúde pública. É preciso destacar os efeitos perniciosos desses produtos à saúde humana e os impactos ambientais negativos do solo, da água e do ar, onde as consequências se manifestam de forma e intensidade diferentes causando um desequilíbrio biológico bem como na saúde dos trabalhadores envolvidos e nas comunidades expostas por tantos anos a essas substâncias comprovadamente nocivas. Essa estratégia considerada "mosquitocêntrica" além de pouco efetiva introduz situações de perigos adicionais para populações já vulnerabilizadas por suas condições de vida.

Neste sentido, consideramos importante incentivar a consciência crítica e concordamos com o grupo que aponta a necessidade de visibilizar os danos ao ambiente e à saúde humana, decorrentes do uso de produtos químicos no controle vetorial, e a prática de revelar às populações vulneráveis, aos profissionais de saúde, incluindo os trabalhadores de saúde pública os efeitos nocivos que hoje são totalmente desconsiderados como as alergias, a imunotoxicidade, os cânceres, distúrbios hormonais, neurotoxicidade, 
dentre outros. Este grupo destaca que na tentativa de eliminar o mosquito estão sendo atingindo os humanos mediante morte lenta, gradual, invisível e ocultada, pois as doenças crônicas causadas por tais produtos aparecem somente no médio e longo prazo, a maioria delas chamadas "idiopáticas", isto é, de causa indefinida (ABRASCO, 2016).

Assim, para entender o que nos faz conviver com esse vetor há décadas, se faz necessário um olhar para além do controle do mosquito pela saúde pública de modo a buscar compreender a complexa rede de elementos que estão inter-relacionados e propor intervenções (eco)sistêmicas de modo a enfrentar as iniquidades sociais tão presentes nos dias atuais para grupos populacionais com maior susceptibilidade para contrair doenças como a Dengue, Chikungunya e Zika e suas consequências. E, neste sentido propomos um o necessário redirecionamento das ações de vigilância em saúde nos municípios para enfrentamento do atual cenário epidemiológico com ações ambientalmente sustentáveis.

\section{REFERÊNCIAS}

ABRASCO. Nota técnica sobre microcefalia e doenças vetoriais relacionadas ao Aedes aegypti: os perigos das abordagens com larvicidas e nebulizações químicasfumacê. 2016. p. 1-11. Disponível em: https://www.abrasco.org.br/site/2016/02/nota-tecnicasobre-microcefalia-e-doencas-vetoriais-relacionadasao-aedes-aegypti-os-perigos-das-abordagens-comlarvicidas-e-nebulizacoes-quimicas-fumace Acesso em: 10 Nov. 2016.

ACHEE, N. L.; GOULD, F.; PERKINS, T. A.; REINER JR, R. C.; MORRISON, A. C.; RITCHIE, S.A.; GUBLER, D. J.; TEYSSOU, R.; SCOTT, T.W. A Critical Assessment of Vector Control for Dengue Prevention. Plos Neglected Tropical Diseases, [s.I.], v. 9, n. 5, p.1-19, Mai. 2015. Public Library of Science (PLoS). Disponível em: http://dx.doi.org/10.1371/journal.pntd.0003655. Acesso em: 10 Nov. 2016.

ARAÚJJ, A. C. P.; NOGUEIRA, D. P.; AUGUSTO, L.G. S. Impacto dos praguicidas na saúde: estudo da cultura de tomate. Revista de Saúde Pública, São Paulo, v. 34, n. 3, p.309-313, jun. 2000. FapUNIFESP (SciELO). Disponivel em: http://dx.doi.org/10.1590/s003489102000000300016. Acesso em: 10 Nov. 2019

AUGUSTO, L. G. S.; SANTOS, S. L.; DIDERICHSEN, $F$. Review of the Aedes aegypti control strategy is needed: chemical warfare or tackling the social determination. WATERLAT-GLOBACIT Research Network, [s.l.], v. 3, p. 62-67, 2016.
BASSI, K. L. Cancer health effects of pesticides: systematic review. Journal of Clinical Oncology, [s.I.], v. 53, n. 10, p. 1704-1711, Out. 2007. Disponível em: https://www.ncbi.nlm.nih.gov/pubmed/17934034. Acesso em: 10 Nov. 2019.

BRAGA, I. A.; VALLE, D. Aedes aegypti: histórico do controle no Brasil. Epidemiologia e Serviços de Saúde, Brasilia, v. 16, n. 2, p. 113-118, Jun. 2007. Disponivel em:

http://scielo.iec.gov.br/scielo.php?script=sci_arttext\&pid $=\$ 1679-49742007000200006$. Acesso em: 10 Nov. 2019.

BRASIL. Instituto Nacional do Câncer. Posicionamento do Instituto Nacional de Câncer José Alencar Gomes da Silva acerca dos Agrotóxicos. Rio de Janeiro: INCA, 2015.

BRASIL. Senado Federal. Decreto $n^{0} 8.675$ de 04/02/1942. Publicação Original [Coleção de Leis do Brasil de 31/12/1942 - vol. 002] (p. 169, col. 1) Disponível em: http://legis.senado.leg.br/norma/414446. Acesso em: 26 março de 2020.

BRASIL. Ministério da Saúde. Secretaria de Vigilância em Saúde. Boletim Epidemiológico Brasília, v.50, n. 13, p.5-18, 2019. Disponivel em: http://portalarquivos2.saude.gov.br/images/pdf/2019/ab ril/30/2019-013-Monitoramento-dos-casos-dearboviroses-urbanas-transmitidas-pelo-Aedespublicacao.pdf. Acesso em: 29 Mai. 2019. 
BRASIL. Ministério da Saúde. Mudança de uso de inseticidas larvicidas e adulticidas na rotina do Programa Nacional de Controle da Dengue. Nota técnica. 2014.

BRASIL. Ministério da Saúde. Secretaria de Vigilância em Saúde. Monitoramento dos casos de dengue, febre de chikungunya e febre pelo vírus Zika até a Semana Epidemiológica 3, 2016. Boletim Epidemiológico, Brasília, v. 47, n. 6. p.1-7, 2016. Disponível em: http://portalsaude.saude.gov.br/images/pdf/2016/feverei ro/04/2016-004---Dengue-SE3.pdf. Acesso em: 10 Nov. 2019.

BRASIL. Ministério da Saúde. Secretaria de Vigilância em Saúde. Nota Técnica n. 109/2010 CGPNCD/DEVEP/SVS/MS. 2010. Disponível em: http://docplayer.com.br/56691710-Nota-tecnica-n-o109-2010-cgpncd-devep-svs-ms.html. Acesso em: 29 Set. 2019.

BRITO C. A. A.; TEIXEIRA, M.G. Increased number of deaths during a chikungunya epidemic in Pernambuco, Brazil. Memórias do Instituto Oswaldo Cruz, Rio de Janeiro, v. 112, n. 9, p. 650-651, Sep. 2017. Disponível em:

http://www.scielo.br/scielo.php?script=sci arttext\&pid= S0074-02762017000900650. Acesso em: 10 Nov. 2019.

CAMPOS M.C,; DOMBROWSKI, J G.; PHELAN, J.; MARINHO, C.R.F.; HIBBERD, M.; CLARCK T.G.; CAMPINO S. Zika might not be acting alone: Using an ecological study approach to investigate potential coacting risk factors for an unusual pattern of microcephaly in Brazil. PLoS ONE, [s.I.], v.13, n. 8, p.11-16, 2018.

https://doi.org/10.1371/journal.pone.0201452. Acesso em: 10 Nov. 2019.

CARLSEN, E.; GIWERCMAN, A.; KEIDING, N.; SKAKKEBAEK, N.E. Evidence for decreasing quality of semen during the past 50 years. Brit Med. J. v, 305, p. 609-613, Sep 1992. Disponível em: https://www.ncbi.nlm.nih.gov/pubmed/1393072. Acesso em: 28 Mar. 2020.

\section{CENTER FOR DESEASE CONTROL AND}

PREVENTION (CDC). Dengue. Transmission. Through Mosquito Bites. 2020 Available in:

https://www.cdc.gov/dengue/transmission/index.html; Acesso em 25 de março de 2020.

CHIARAVALLOTI, V.B; MORAIS M.S; CHIARAVALLOTI-NETO, F.; CONVERSANI, D.T.; FIORIN, A.M.; BARBOSA, A. A. C.; FERRAZ, A. A.; Avaliação sobre adesão às práticas preventivas do dengue: 0 caso de Catanduva, São Paulo, Brasil. Cadernos de Saúde Pública, Rio de Janeiro, v. 18, n. 5, p.1321-1329, set/out. 2002. Disponível em: https://pdfs.semanticscholar.org/01ff/60130a3c18d7864 d42b7693e537cfa0bdfdd.pdf. Acesso em 25 Mar. 2020.

COSTA, A. M. A determinação social da microcefalia/zika no Brasil. WATERLAT-GOBACIT Network Working Papers. Thematic Area Series SATAGSA - TA5 - Water and Health - [s.I.], v. 3, n. 9 , p.44-61, Dec. 2016. ISSN 2056-4856 (Print). ISSN 2056-4864 (Online). Disponível em:

http://waterlat.org/WPapers/WPSATGSA39.pdf. Acesso em: 25 Mar. 2020.

FONTENELE, E. G. P.; MARTINS, M. R. A.; QUIDUTE, A. R. P.; MONTENEGRO JÚNIOR, R. M.

Contaminantes ambientais e os interferentes endócrinos. Arquivos Brasileiros de Endocrinologia \& Metabologia, São Paulo, v. 54, n. 1, p.6-16, Feb. 2010. Disponível em:

http://www.scielo.br/scielo.php?script=sci_arttext\&pid= S0004-27302010000100003. Acesso em: 10 Nov. 2016.

FRANCO, O. História da Febre Amarela no Brasil. Rio de Janeiro: Superintendência de Campanhas de Saúde Pública. Ministério da Saúde; 1969.

FRIEDRICH, K. Desafios para a avaliação toxicológica de agrotóxicos no Brasil: desregulação endócrina e imunotoxicidade. Revista Vigilância Sanitária em Debate [s.I.], v. 1, n. , p. 2-15, Mai. 2013. Disponível em:

https://www.researchgate.net/publication/307757879_D esafios_para_a_avaliacao_toxicologica_de_agrotoxicos _no_Brasil_desregulacao_endocrina_e_imunotoxicidad e_Challenges_to_toxicological_evaluation_of_pesticide s_in_Brazil_endocrine_disruption_and_immunot. Acesso em: 15 set. 2019.

FUNDAÇÃO NACIONAL DE SAÚDE. Dengue instruções para pessoal de combate ao vetor: manual de normas técnicas. - 3. ed., rev. - Brasília, 2001b.

FUNDAÇÃO NACIONAL DE SAÚDE (Brasil). Plano de intensificação das ações de controle do dengue. Brasilia, DF, 2001a.

FUNDAÇÃO NACIONAL DE SAÚDE (Brasil). Plano Diretor de Erradicação do Aedes aegypti no Brasil: versão atualizada em: 01 de mar. Brasília, DF, 1996.

FUNDAÇÃO NACIONAL DE SAÚDE (Brasil).

Programa Nacional de Controle da Dengue: instituído em 24 de julho de 2002. Brasília, DF, 2002.

GHISELLI, G; JARDIM, W . F. Interferentes endócrinos no ambiente. Química Nova, São Paulo, v. 30, n. 3, p.695-706, Mai/Jun. 2007. Disponível em: http://www.scielo.br/scielo.php?script=sci_arttext\&pid= S0100-40422007000300032. Acesso em: 27 Mar. 2020. 
GRISOLIA, C. K. Agrotóxicos: mutações, câncer e reprodução. Brasília, DF: Universidade de Brasília, 2005. 392p.

GUEDES D. R. D.; PAIVA, M.H. S.; DONATO, M. M. A.; BARBOSA, P. P.; KROKOVSKY, L.; ROCHA, S. W. S.; SARAIVA, K. L. A.; CRESPO, M. M.; BARBOSA, R. M. R.; OLIVEIRA, C. M. F. et al. Zika virus replication in the mosquito Culex quinquefasciatus in Brazil. Emerg Microbes Infect, v.6, n.8, Ago. 2017.

Disponível em: https://www.ncbi.nlm.nih.gov/pubmed/28790458. Acesso em: 25 Mar.2020.

GUYTON, K. Z. ; LOOMIS, D.; GROSSE, Y.; GHISSASSI, F.E.; BENBRAHIM-TALLA, L.; GUHA, N. Carcinogenicity of tetrachlorvinphos, parathion, malathion, diazinon, and gluphosate. The Lancet oncology, v. 16, n. 5, p. 490-491, May. 2015. Disponível em: https://www.thelancet.com/journals/lanonc/article/PIIS1 470-2045(15)70134-8/fulltext. Acesso em 29 Mar. 2019.

GURGEL, I. G. D. Saúde dos trabalhadores que atuam no controle de endemias vetoriais. In: AUGUSTO, L.G.S.; CARNEIRO, R.M.; MARTINS, P. H. Org. Abordagem ecossistêmica em Saúde: Ensaios para 0 controle de dengue. Recife: Editora Universitária; 2005. p. 227-234.

\section{INTERNATIONAL AGENCY FOR RESEARCH ON}

CANCER (IARC) Malathion. IARC Monographs Volume 112: evaluation of five organophosphate inseticides and herbicides, 2015. Disponivel:

https://www.iarc.fr/en/media-

centre/iarcnews/pdf/Monograph Volume112.pdf.

Acesso em 29 Mar. 2019.

LEÃO M. B. C.; PAVÃO, A.C. Molecular Orbital Análises of Chemical Carcinogens, International Journal of Quantum Chemistry, [s.I.], n. 6, p. 323-328, Dec.1996. Disponível em:

https://onlinelibrary.wiley.com/doi/epdf/10.1002/\%28SIC 1\%291097-

461X\%281997\%2962\%3A3\%3C323\%3A\%3AAIDQUA11\%3E3.0.CO\%3B2-A Acesso em: 29 Mar. 2019.

LEFEVRE A. M. C., RIBEIRO, A. F.; MARQUES, G. R. A. M.; SERPA, L. L. N.; LEFĖVRE, F. Representações sobre dengue, seu vetor e ações de controle por moradores do Município de São Sebastião, Litoral Norte do Estado de São Paulo, Brasil. Cadernos de Saúde Pública, Rio de Janeiro, v. 23, n. 7, p.16961796, Jul. 2007. Disponível em: http://www.scielo.br/scielo.php?script=sci_arttext\&pid= S0102-311X2007000700022. Acesso em: 29 Mar 2019.
LOWY, llana. Representação e intervenção em saúde pública: vírus, mosquitos e especialistas da Fundação Rockefeller no Brasil. Hist. cienc. saude-Manguinhos, Rio de Janeiro , v. 5, n. 3, p. 647-677, Feb. 1999. Disponível em:

http://www.scielo.br/scielo.php?script=sci_arttext\&pid= S0104-59701999000100006\&lng=en\&nrm=iso. Acesso em: 29 Mar. 2019.

MARICATO, E. As cidades, o mosquito e as reformas. WATERLAT-GOBACIT Network Working Papers. Thematic Area Series SATAGSA - TA5 - Water and Health, [s.I.], v. 3, n. 9, p. 30-37, Dec. 2016. ISSN 20564856 (Print). ISSN 2056-4864 (Online) Disponível em: http://waterlat.org/WPapers/WPSATGSA39.pdf. Acesso em: 25 Mar. 2020.

NEVES, D. P. Parasitologia Humana. 12 ed. São Paulo: Atheneu, 2016.

OPAS. Manual de Vigilância da Saúde de Populações Expostas a Agrotóxicos. Ministério da Saúde, Secretaria de Vigilância Sanitária. Brasília: OPAS/OMS, 1996.

PIRES, D. X.; CALDAS, E. D.; RECENA, M. C. P. Intoxicações provocadas por agrotóxicos de uso agrícola na microrregião de Dourados, Mato Grosso do Sul, Brasil, no período de 1992 a 2002. Cadernos de Saúde Pública, Rio de Janeiro, v. 21, n. 3, p. 804-14, Mai/Jun. 2005. Disponível em:

http://www.scielo.br/scielo.php?script=sci_arttext\&pid= S0102-311X2005000300014. Acesso em: 29 Mar. 2019.

RIGOTTO, R. M; AGUIAR, A. C. P. Invisibilidade ou invisibilização dos efeitos crônicos dos agrotóxicos à saúde? Desafios à ciência e às políticas públicas. 2015. E-book. Disponível em:

http://capacidadeshumanas.org/oichsitev3/wpcontent/uploads/2018/10/03_agrotoxicos-final.pdf

Acesso em 15 Set. 2019.

ROMANO, R. M.; ROMANO, M. A.; MOURA, M. O.; OLIVEIRA, C. A. A exposição ao glifosato-Roundup causa atraso no início da puberdade em ratos machos. Brazilian Journal of Veterinary Research and Animal Science , [s.l.], v. 45, n. 6, p. 481-487. 2008.

SÁ-SILVA, J. R.; ALMEIDA, C. D.; GUINDANI, J. F. Pesquisa documental: pistas teóricas e metodológicas. Revista Brasileira de História \& Ciências Sociais, [s.I.], n. 1. p.1-14, 2009. Disponivel em: https://periodicos.furg.br/rbhcs/article/view/10351/0. Acesso em: 15 Set. 2019.

SANTOS, Solange Laurentino dos. Avaliação das ações de controle de dengue: aspectos críticos e percepção da população. 2003. Dissertação (Mestrado 
em Saúde Pública) - Centro de Pesquisas Aggeu Magalhães, Fundação Oswaldo Cruz, Recife, Pernambuco, 2003.

SANTOS, S. L.; AUGUSTO, L. G. S. Dengue: uma avaliação das ações do programa de controle.

Cadernos Saúde Coletiva, Rio de Janeiro, v. 13, n. 1, p.263-280, 2005. Disponível em:

http://www.cadernos.iesc.ufrj.br/cadernos/images/csc/2 005_1/artigos/Cad20051_solange.pdf. Acesso em: 15 Set. 2019.

SANTOS, S. L.; CABRAL, A. C. S. P.; AUGUSTO, L. G. S. Conhecimento, atitude e prática sobre dengue, seu vetor e ações de controle em uma comunidade urbana do Nordeste. Ciência e Saúde Coletiva, Rio de Janeiro, v. 16, p. 1319-1330, 2011. Supl 1. Disponível em: http://www.scielo.br/scielo.php?script=sci_arttext\&pid= S1413-81232011000700066. Acesso em: 15 Set. 2019.

SANTOS, S. L.; SILVA, M. B. C. E. ; CABRAL, A. C. S. P. ; GONCALVES, G. M. S. ; AUGUSTO, L. G. S.

Percepção sobre o controle da dengue: uma análise a partir do discurso coletivo. Movimentos Sociais e Dinâmicas Espaciais, Recife, v. 4, p. 115-130, 2015. Disponível em:

https://periodicos.ufpe.br/revistas/revistamseu/article/vi ew/229883. Acesso em: 25 Mar. 2020.

SANTOS, V.M. R.; DONNICI, C.L. DACOSTA, J. B. N.; CAIXEIRO, J. M. R. Compostos organofosforados pentavalentes: histórico, métodos sintéticos de preparação e aplicações como inseticidas e agentes antitumorais. Química Nova, São Paulo, v. 30, n. 1, p.159-170, Jan/Fev. 2007. Disponível em: http://www.scielo.br/scielo.php?script=sci_arttext\&pid= S0100-40422007000100028. Acesso em: 15 Set. 2019.

SOBRAL, M. F. F.; SOBRAL, A. I. G. P. Casos de dengue e coleta de lixo urbano: um estudo na Cidade do Recife, Brasil. Ciência \& Saúde Coletiva, Rio de Janeiro, v. 24, n. 3, p.1075-1082, Mar. 2019. Disponível em:

http://www.scielo.br/scielo.php?script=sci_arttext\&pid= S1413-81232019000301075\&lng=en\&nrm=iso. Acesso em: 15 Set. 2019.

SOLOMON, G. M.; SCHETTLER, T. Environment and Health: Endocrine disruption and potential human health implications. Canadian Medical Association of Journal, [s.I.], v. 163, n. 11, p.1471-1476, Nov. 2000. Disponível em: https://www.researchgate.net/publication/299245616_E nvironment_and_health_6_Endocrine_disruption_and_ potential_human_health_implications. Acesso em: 15 Set. 2019.

SOUZA, W.V.; ALBUQUERQUE, M. F. P. M.; VAZQUEZ, E.; BEZERRA, L. C. A.; MENDES, A. D. C. G.; LYRA, T. M.; ARAUJO, T. V. B.; OLIVEIRA. A. L.
S.; BRAGA, M. C.; XIMENES, R. A. A.; MIRANDAFILHO, D. B.; CABRAL SILVA, A. P. S.; RODRIGUES. L.; MARTELLI, C. M. T. Microcephaly epidemic related to the Zika virus and living conditions in Recife, Northeast Brazil. BMC Public Health . [s.I.], v. 8, n. 1, p.130- 137. Jan. 2018. Disponível em: https://www.ncbi.nlm.nih.gov/pubmed/29329574. Acesso em: 15 Set. 2019.

TAUIL PL. Urbanização e ecologia da dengue. Cadernos de Saúde Pública, Rio de Janeiro, v. 17, p. 99-102, 2001. (Supl). Disponível em: http://www.scielo.br/scielo.php?pid=S0102311X2001000700018\&script=sci_abstract\&tlng=pt. Acesso em 15 Set. 2019.

VALLE, D.; Controle químico de Aedes aegypti, Resistência a Inseticidas e alternativas. In: VALLE, D, PIMENTA DN, CUNHA, R. V. Dengue teorias e práticas. 2015. p. 75-92.

WORLD HEALTH ORGANIZATION (WHO). Executive Board requests resolution on a comprehensive approach to global vector control. [acesso em: 29/05/2019]. Disponível em https://www.who.int/neglected_diseases/news/Resoluti on_on_comprehensive_approach_

to_global_vector_control/en/.2019. Acesso em: 15 Set. 2019.

WORD HEALTH ORGANIZATION PESTICIDE EVALUANTION SCHEME (WHOPES). WHO pesticide evaluation scheme: 50 years of global leadership, 2010. Disponivel em: <http:// apps.who.int/IRIS/HANDLE/10665/44305. Acesso: 25 Ago. de 2016.

SILVA, T.R.B., COSTA, P.F.F, SANTOS, S. L. Perigos no uso de agrotóxicos pela saúde pública no controle vetorial do Aedes Aegypti... 